\title{
Testosterone metabolism of equine single CYPs of the 3A subfamily compared to the human CYP3A4
}

\author{
S. Vimercati ${ }^{1}$, M. Büchi ${ }^{1}$, J. Zielinski, N. Peduto, M. Mevissen * \\ Veterinary Pharmacology and Toxicology, Department of Clinical Research and Veterinary Public Health, Vetsuisse Faculty, University of Bern, Laenggassstrasse 124, 3012 Bern, Switzerland
}

\section{A R T I C L E I N F O}

\section{Article history:}

Received 9 December 2016

Received in revised form 6 February 2017

Accepted 20 February 2017

Available online 24 February 2017

\section{Keywords:}

Cytochrome P450 enzyme

Horse

Metabolism

Hydroxytestosterone

\begin{abstract}
A B S T R A C T
Cytochrome P450 enzymes (CYPs) are responsible for the phase I metabolism of drugs, xenobiotics and endogenous substances. Knowledge of single CYPs and their substrates is important for drug metabolism, helps to predict adverse effects and may prevent reduced drug efficacy in polypharmacy. In this study, three equine isoenzymes of the 3A subfamily, the equine flavoprotein NADPH-P450 oxidoreductase (POR), and the cytochrome $b 5$ (CYB5) were cloned, sequenced and heterologously expressed in a baculovirus expression system. Testosterone, the standard compound for characterization of the human CYP3A4, was used to characterize the newly expressed equine CYPs. The metabolite pattern was similar in equine and the human CYPs, but the amounts of metabolites were isoform-dependent. All equine CYPs produced 2-hydroxytestosterone (2-OHTES), a metabolite never described in equines. The main metabolite of CYP3A4 6 3 -hydroxytestosterone (6 $63-$ OH-TES) was measured in CYPs $3 \mathrm{~A} 95$ and $3 \mathrm{~A} 97$ with levels close to the detection limit. Ketoconazole inhibited 2-OH-TES in the human CYP3A4 and the equine CYP3A94 and CYP3A97 completely, whereas a 70\% inhibition was found in CYP3A95. Testosterone 6 $\beta$ - and 2-hydroxylation was significantly different in the equine CYPs compared to CYP3A4. The expression of single equine CYPs allows characterizing drug metabolism and may allow prevention of drug-drug interactions.
\end{abstract}

(c) 2017 Elsevier Ltd. All rights reserved.

\section{Introduction}

Cytochrome P450 enzymes (CYPs) are haemoproteins and act as monooxygenases responsible for the phase I metabolism of drugs and toxins, and for the biotransformation of endogenous substances, e.g. cholesterol and steroids (Guengerich, 2003; Li and Apte, 2015). Human CYPs are divided into multiple families; however, only CYP families one, two, and three have been demonstrated to be mainly responsible for drug metabolism in humans.

Human CYP3A represents the most important subfamily involved in the metabolism of $>50 \%$ of therapeutic drugs (Wilkinson, 2005; Werk and Cascorbi, 2014). The characterization of the isoenzymes is important to avoid adverse effects or lack of response in therapy with

Abbreviations: POR, NADPH P450 oxidoreductase; CYPs, cytochrome P450 enzymes; CYB5, cytochrome b5; Sf9, insect cells of Spodoptera frugiperda; HPLC, high-pressure liquid chromatography; NADPH, nicotinamide adenine dinucleotide phosphate; NDSB, non-detergent sulfobetaine; TES, testosterone; 2-OH-TES, 2-hydroxytestosterone; 6ßOH-TES, 6ß-hydroxytestosterone; 11ß-OH-TES, 11ß-hydroxytestosterone.

* Corresponding author at: Division of Veterinary Pharmacology and Toxicology, Department of Clinical Research and Veterinary Public Health, Vetsuisse Faculty, University of Bern, Laenggassstrasse 124, CH-3012 Bern, Switzerland.

E-mail addresses: sara.vimercati@vetsuisse.unibe.ch (S. Vimercati),

jana.zielinski@vetsuisse.unibe.ch (J.Zielinski),nadja.peduto@vetuisse.unibe.ch

(N. Peduto), meike.mevissen@vetsuisse.unibe.ch (M. Mevissen).

${ }^{1}$ Authors contributed equally to the work. polypharmacy due to the ability of inhibition or induction of CYPs by various substances. Prior to drug administration, pharmacokinetics and pharmacodynamics should be evaluated since many drug-drug interactions have been observed (Yu et al., 2014; Bohnert et al., 2016).

Human CYPs have been well characterized, but little is known about CYPs in horses. The genetic similarity of the human CYP3A subfamily, especially the isoform CYP3A4 with the isoenzymes of the equine CYP3A subfamily has been reported to be higher than $90 \%$. The number of isoenzymes of this CYP subfamily differs significantly between human and horses (Schmitz et al., 2010a). Despite the similarity in mRNA, it is important to note that several studies have shown species differences in functionality of CYPs (Chauret et al., 1997; Nebbia et al., 2003; Fink-Gremmels, 2008; Knych et al., 2009; Knych et al., 2010). Our group identified seven equine genes of the subfamily CYP3A, namely CYP3A89, CYP3А93, CYP3A94, CYP3A95, CYP3A96, CYP3A97 and CYP3A129 (Schmitz et al., 2010a, 2010b). To date, in vitro studies regarding functional information related to these isoenzymes are limited, and sparse characterization of CYPs 3A89, 3A94, 3A95, 3A96, and 3A97 has been done (Knych et al., 2010; Schmitz et al., 2014; Dettwiler et al., 2014). Several functional studies on equine microsomal protein from different tissues including liver and primary equine liver cells are available (Schmitz et al., 2008; Capponi et al., 2009; Mössner et al., 2011; Stefanski et al., 2013; Zielinski and Mevissen, 2015), but these studies include all CYP-isoenzymes. The results of such studies are 
always a combination of different metabolism reactions. Hence, liver microsomes do not allow conclusions of CYP isoenzymes involved in the metabolism of a compound. In contrast, heterologous expression of single CYPs allows identifying isoenzymes involved in drug metabolism and predicting possible drug-drug interactions. Besides CYPs, flavoprotein NADPH P450 oxidoreductase (POR) and cytochrome b5 (CYB5) are essential for the function of the CYPs. POR is responsible for the transport of electrons from the reductive NADPH to the CYP. CYB5 is vital for some CYPs, e.g. the human CYP3A4 as an electron transporter (Schenkman and Jansson, 2003; Pandey and Flück, 2013). We recently reported that co-expression of equine POR increased CYP expression and activity in Chinese hamster fibroblasts (Dettwiler et al., 2014). CYB5 was found in different equine tissues at varying levels with higher amounts in the tissue of the upper airways than in the horse liver (Tydén et al., 2008). Besides the specific amounts of each enzyme, the ratios of POR:CYP:CYB5 differ between these tissues and appear to be important for the CYP activity (Venkatakrishnan et al., 2000; Tyden et al., 2008; Knych et al., 2010). Therefore, we hypothesized that the equine CYB5 is also crucial for the equine CYP3A isoforms.

In veterinary medicine, little is known about substrate specificity in horses. Testosterone has been used to investigate the function of CYPs in various animal species (Khalil et al., 2001; Shou et al., 2003; van Waterschoot and Schinkel, 2011; Yao et al., 2011). The U.S. Food and Drug Administration recommends testosterone and midazolam as suitable substrates to characterize CYP3A4/5 (FDA, 2006). Testosterone 6 $\beta-$ hydroxylation has been demonstrated to differ between humans versus horses, cats, and dogs (Chauret et al., 1997; Fink-Gremmels, 2008; Knych et al., 2010; Scarth et al., 2011; Zielinski and Mevissen, 2015). Despite a few studies on functional characterization of equine isoenzymes of the CYP3A family (Knych et al., 2010; Schmitz et al., 2014; Nakayama et al., 2016), limited information about the single CYPs of the subfamily 3A, POR, and CYB5 in horses is available. To date, no data on the effect of different POR:CYP:CYB5 ratios of CYP3A94, CYP3A95 and CYP3A97 are available.

This study was designed to develop an in vitro system using a baculovirus-based transduction technique for the expression of single equine CYPs of the $3 \mathrm{~A}$ subfamily and their auxiliary proteins, POR and CYB5, in cells of the insect Spodoptera frugiperda (Sf9). These cells are often used for the expression of CYPs because they produce high yields of the favoured protein and are able to conduct many eukaryotic posttranslational modifications (Demain and Vaishnav, 2009; Thompson et al., 2016). The respective equine CYPs were characterized using the standard compound testosterone as substrate as well as standard inhibitor for the CYP3A subfamily ketoconazole. Pharmakokinetic analyses with testosterone were performed for the equine CYPs and compared to the human CYP3A4, a CYP that has been well characterized. This comparison is also of interest because many drugs used in human therapy are also used in equine therapy.

\section{Materials and methods}

\subsection{Reagents}

Sf-900 II SFM and Grace insect cell medium, fetal bovine serum (FBS), phosphate-buffered saline (PBS), L-glutamine, insulin-transferrin-selenium, penicillin, and streptomycin were purchased from Gibco (Carlsbad, CA, USA). TRIzol reagent was obtained from Invitrogen (Carlsbad, CA, USA). KOD Hot Start DNA Polymerase, BacMagic-3 DNA Kit, insect gene juice transfection reagent, chemiluminescent HRP substrate, and sucrose were obtained from Merck Millipore AG (Zug, Switzerland). Ferric citrate, 5 -aminolevulinic acid hydrochloride (5-ALA), bovine albumin (BSA), $\beta$ $\mathrm{NADH}$, sodium dithionite, testosterone, and the cytochrome $c$ reductase (NADPH) assay kit were purchased from Sigma-Aldrich Chemie GmbH (Buchs, Switzerland). Page ruler plus pre-stained protein ladder, halt protease inhibitor cocktail, and EDTA were obtained from Thermo Fisher Scientific AG (Reinach, Switzerland). Mouse monoclonal antibody against
HIS-tag was bought from Enzo Life Sciences (ELS) AG (Lausen, Switzerland). Rabbit polyclonal antibody against rat POR was obtained from Abcam (Cambridge, UK). Rabbit polyclonal antibody against human cytochrome $b 5$ and mouse monoclonal anti-human CYP3A4 antibody were purchased from Santa Cruz Biotechnology Inc. (LabForce AG, Muttenz, Switzerland). Horseradish peroxidase (HRP) labeled secondary antibodies - donkey anti-mouse and goat anti-rabbit - were purchased from Novex (Thermo Fisher, Dreieich, Germany). NADPH Regenerating System was obtained from Promega AG (Dübendorf, Switzerland). Corning Supersomes human CYP3A4 + reductase + b5 were obtained from Corning (Amsterdam, The Netherlands). Methanol and acetonitrile, both HPLC grade, were obtained from EGT Chemie (Tägerig, Switzerland). The internal standard testosterone 17-propionate (TES-17-P) was purchased from Lipomed AG, (Arlesheim, Switzerland). The stock solutions of testosterone and androstenedione were obtained from Sigma-Aldrich Chemie Gmbh (Buchs, Switzerland). 6ß-hydroxytestosterone was purchased from Lipomed AG (Arlesheim, Switzerland), 11ßhydroxytestosterone was obtained from Chemie Brunschwig AG (Basel, Switzerland), $2 \alpha$-hydroxytestosterone, $2 \beta$-hydroxytestosterone, $16 \alpha$ hydroxytestosterone were obtained from Biozol (Eching, Germany), 16ß-hydroxytestosterone (Steraloid, USA). Ketoconazole was purchased from Sigma (Buchs, Switzerland).

Glycerol was purchased from Gerbu Biotechnik GmbH (Heidelberg, Germany). NADPH regenerating system was obtained from Promega (Dübendorf, Switzerland), testosterone was purchased from Lipomed AG (Arlesheim, Switzerland).

\subsection{RNA isolation and $c D N A$ synthesis}

Equine liver samples were collected from three healthy horses (19 year old warmblood mares and a three year old FranchesMontagnes mare) without recent history of drug administration at the abattoir. RNA isolation, cDNA synthesis, and RT-PCR were conducted as described elsewhere (Schmitz et al., 2008a). Briefly, small liver pieces were homogenized in TRIzol Reagent, and RNA was isolated using an RNeasy Kit (Qiagen, Hilden, Germany). Reverse transcription was conducted using QuantiTect Reverse Transkription kit (Qiagen).

\subsection{Amplification, cloning and sequencing of equine CYPs, POR, and CYB5}

The DNA of each gene was amplified using KOD Hot Start DNA Polymerase and cloned into the multiple cloning sites of separate $\mathrm{pIEx} / \mathrm{Bac}-1$ expression vectors (Merck) using the In-Fusion HD Cloning Kit of Clontech (Mountain View, CA, USA). Primers were designed using Primer3Plus software (http://www.bioinformatics.nl/cgi-bin/ primer3plus/primer3plus.cgi) or were designed manually and a HIStag was inserted at the 3 '-end of the CYP3A isoforms in order to evaluate protein expression using immunoblotting. The primers used are given in Table 1 . The DNA was sequenced by an automatic ABI 3730 capillary sequencer (Applied Biosystems, Carlsbad, CA, USA) and compared to the equine sequences of the EMBL database using Sequencher 5.1 (GeneCodes, Ann Arbor, MI, USA) and BLASTed against the equine chromosome 8 and 13 (NCBI BLAST search, http://blast.ncbi.nlm.nih.gov/ Blast.cgi). Subsequently, the plasmids were amplified and purified, and the DNA was sequenced again.

\subsection{Expression and isolation of microsomes}

Sf9 cells were used for heterologous expression of the equine CYPs, POR, and CYB5. The cells were cultivated in Sf-900 II SFM and Grace Insect Medium with $10 \%$ FBS, L-glutamine, penicillin, and streptomycin in suspension culture. The genes of the respective equine CYPs of the $3 \mathrm{~A}$ subfamily were cloned into PIEX/Bac-1 vector, and separately incubated with BacMagic DNA to obtain the plasmid used to transduct Sf9 cells, as described in the manufacturer's protocol (Merck Millipore AG, Zug, 
Table 1

Primer pairs.

\begin{tabular}{|c|c|c|}
\hline No. & Primer name & Sequence $5^{\prime}-3^{\prime}$ \\
\hline \multirow[t]{2}{*}{1} & POR_forward primer & TATTTACAATCCATGGGGGACTCCAACATGGACG \\
\hline & POR_reverse primer & CTCCAGCTTGCCATGGCTACTAGCTCCACACGTCCAGCG \\
\hline \multirow[t]{2}{*}{2} & CYB5_forward primer & TATTTACAATCCATGGCCGAGCAGTCGGACAA \\
\hline & CYB5_reverse primer & CTCCAGCTTGCCATGGCTATTAGTCTTCCGCCGTGTAGATG \\
\hline \multirow[t]{2}{*}{3} & CYP3A94_forward primer & TATTTACAATCCATGGACCTAATCCCAAACTTTTCC \\
\hline & CYP3A94_reverse primer & СTCCAGCTTGCCATGGCTACTAATGATGATGATGATGATGGCCTCCTGAGCCTCCTGAGGCTCCATTCCAGGTACCA \\
\hline \multirow[t]{2}{*}{4} & CYP3A95_forward primer & TATTTACAATCCATGGACCTGATCCCAAGCCTTTC \\
\hline & CYP3A95_reverse primer & CTCCAGCTTGCCATGGCTACTAATGGTGATGGTGATGATGGCCTCCTGAGCCTCCTGAGGCTCCACTCATGGTCCC \\
\hline \multirow[t]{2}{*}{5} & CYP3A97_forward primer & TATTTACAATCCATGGACCTGATCCCCAACTTTTC \\
\hline & CYP3A97_reverse primer & СTCCAGCTTGCCATGGCTACTAGTGGTGGTGATGGTGATGATGGTGGTGATGGCCTCCTGAGCCTCCTGAGGCTCCAGTCATGGTCCCA \\
\hline
\end{tabular}

Switzerland). After two amplification rounds in Sf9 cells, the viral titer was determined with a plaque assay. Briefly, insect cells in exponential phase were seeded in a 6-well plate and were incubated with $100 \mu \mathrm{l}$ of different recombinant baculovirus dilutions for $1 \mathrm{~h}$ at room temperature. After the removal of the baculovirus, $2 \mathrm{ml}$ of insect cell medium containing $1 \%$ of sterile SeaPlaque Agarose (Lonza, Basel, Switzerland) were added to each well. Plaques from baculovirus infected cells were counted in the agarose monolayer after four days of incubation at $27 \mathrm{de}-$ grees and the viral titer was calculated as described in the manufacturer's protocol. Optimal multiplicity of infection (MOI) and the best cell collection time were determined by infecting the cells using different MOI from 0.05 to 10 plaque-forming units/cell and post-infection harvest times from $24,48,72$ and $96 \mathrm{~h}$ at $27{ }^{\circ} \mathrm{C}$ without $\mathrm{CO}_{2}$. The medium was supplemented with insulin-transferrin-selenium (1.7 $\mu \mathrm{M}$ insulin, $69 \mu \mathrm{M}$ transferrin, and $39 \mathrm{nM}$ sodium selenite), $0.02 \mathrm{mM}$ ferric citrate, and $0.2 \mathrm{mM}$ 5-Aminolevulinic acid hydrochloride (5-ALA) for the transduction. For harvesting, the cells were washed with phosphate buffered saline (PBS) and resuspended in $10 \mathrm{mM}$ HEPES, pH 7.8, with $250 \mathrm{mM}$ sucrose, $1 \mathrm{mM}$ EGTA, $25 \mathrm{mM}$ potassium chloride, and proteinase inhibitor using the concentration proposed by the manufacturer. The resuspension was followed by repeated $30 \mathrm{~s}$ sonication cycles on ice (Sonifier W-450 D, Branson, Danbury, CT, USA). Three centrifugation rounds were carried out to isolate membranes of the endoplasmic reticulum: $1^{\prime} 000 \mathrm{~g}$ for $10 \mathrm{~min}, 12^{\prime} 000 \mathrm{~g}$ for $15 \mathrm{~min}$, and $154^{\prime}$ $000 \mathrm{~g}$ for $62 \mathrm{~min}$ at $4{ }^{\circ} \mathrm{C}$. The ultracentrifuge (CP100NX, rotor: TH641) was purchased from Hemotec (Gelterkinden, Switzerland). The same procedure was done with non-transduced cells, which were used as negative control. The microsomal pellet was resuspended in $10 \mathrm{mM}$ HEPES, pH 7.8, with $250 \mathrm{mM}$ sucrose, $1 \mathrm{mM}$ EGTA, $25 \mathrm{mM}$ potassium chloride, with an additional 20\% (v/v) glycerol and $50 \mathrm{mM}$ NDSB211, and was homogenized using a glass potter (neoLab, Heidelberg, Germany). The microsomal protein concentration was measured with a spectrophotometer (NanoDrop 2000c; Thermo Fisher Scientific AG, Reinach, Switzerland). Bovine serum albumin (BSA) was used to build a standard curve to quantify the microsomal protein samples. Protein identification was carried out by Western blotting. It was conducted using a $12 \%$ acrylamide-gel, followed by a blocking in phosphate-buffered saline $0.1 \%$ Tween-20 (PBST) with 5\% skim milk for $2 \mathrm{~h}$. The membranes were incubated overnight with a rabbit antibody against POR (1:4000), a rabbit antibody against CYB5 (1:600), and a mouse anti-HIS antibody (1:2000) to detect the CYPs, followed by a $2 \mathrm{~h}$ incubation with the respective secondary antibodies (goat anti-rabbit, and donkey antimouse, 1:10,000) labeled with horseradish peroxidase.

\subsection{Spectrophotometric analysis of CYP, POR, and CYB5}

The amount of cytochrome P450 was measured using a spectrophotometer as described earlier (Omura and Sato, 1964). In brief, $1 \mathrm{mg} / \mathrm{ml}$ microsomal protein was reduced with a few milligrams of sodium dithionite. After determining the base line, the samples were aerated with $\mathrm{CO}$ for $1 \mathrm{~min}$ and were measured again. Once the difference between these two values was obtained, the amount of CYP was calculated based on the CYP coefficient of $91 \mathrm{mM}^{-1} \mathrm{~cm}^{-1}$. To determine the amount of POR, cytochrome $c$ reductase activity was measured according to the manufacturer's protocol (cytochrome $c$ reductase assay kit, Sigma, Switzerland). Briefly, cytochrome $c$ from equine heart muscle was reduced by POR and the spectra was measured at $550 \mathrm{~nm}$ for $1 \mathrm{~min}$. The molar extinction coefficient for reduced cytochrome $c$ is $21 \mathrm{mM}^{-1} \mathrm{~cm}^{-1}$. Since CYB5 does not bind CO, it was reduced with $5 \mu \mathrm{l}$ of $20 \mathrm{mM} \beta-\mathrm{NADH}$. The baseline of oxidized CYB5 $(1 \mathrm{mg} / \mathrm{ml})$ was measured at $410 \mathrm{~nm}$ followed by the measurement of the reduced CYB5 at $425 \mathrm{~nm}$. The difference between the oxidized and reduced CYB5 was used to calculate the concentration of CYB5. The extinction coefficient $185 \mathrm{mM}^{-1} \mathrm{~cm}^{-1}$ for reduced CYB5 was used to calculate the CYB5 concentration (Gan et al., 2009).

\subsection{Enzymes activity and kinetics}

\subsubsection{Testosterone metabolism}

To analyze possible testosterone biotransformation, human CYP3A4 supersomes and all investigated equine microsomal CYP proteins, as well as various amounts of equine POR and CYB5 were pre-incubated for $5 \mathrm{~min}$ at $37{ }^{\circ} \mathrm{C}$ in $100 \mathrm{mM}$ PBS ( $\mathrm{pH} \mathrm{7.4)}$ ) using the NADPH regenerating system. The reaction was started by adding $100 \mu \mathrm{M}$ testosterone to the mixture. POR to CYP to CYB5 ratios of 4 [pmol]:1 [pmol]:1 [pmol] in a total volume of $1 \mathrm{ml}$ were used, and several time points from 0 to $8 \mathrm{~h}$ were examined to determine linear conditions. The optimal incubation time for the testosterone metabolism was investigated for all three equine CYPs. Testosterone $6 \beta$ and 2-hydroxylation kinetics were performed for the equine CYPs and CYP3A4 using different concentrations ranging from 1 to $100 \mu \mathrm{M}$ and $1-200 \mu \mathrm{M}$, respectively. Intrinsic clearance, the rate of metabolism for a given drug concentration, and the maximum clearance were analyzed (Table 2). Maximum clearance shows the highest clearance that can be obtained while the concentration of the substrate increases before reaching enzyme saturation.

All testosterone incubations were stopped by adding ice-cold acetonitrile. The internal standard testosterone 17-propionate was added before centrifugation at $16^{\prime} 600 \mathrm{~g}$ for $10 \mathrm{~min}$. $200 \mu \mathrm{l}$ were used for subsequent analysis. The stock solutions of testosterone as well as a panel of possible metabolites including $6 \beta$-hydroxytestosterone, $11 \beta$ hydroxytestosterone, $2 \alpha$-hydroxytestosterone, $2 \beta$-hydroxytestosterone, $16 \alpha$-hydroxytestosterone, 16 3 -hydroxytestosterone and androstenedione were diluted in methanol. The diluent concentration in all enzyme activity assays did not exceed $0.1 \%(v / v)$.

\subsubsection{Inhibition assay}

Ketoconazole, a known human CYP3A inhibitor (Novotná et al., 2014), previously shown to influence equine microsomal activity (Zielinski and Mevissen, 2015), was used to study the inhibition of the metabolism of TES in all three investigated equine CYPs. The inhibition rates of $63-\mathrm{OH}-\mathrm{TES}$ (for CYP3A94) and 2-OH-TES (for all three equine CYPs) were compared to CYP3A4.

The microsomes were pre-incubated with $1 \mu \mathrm{M}$ ketoconazole at $37{ }^{\circ} \mathrm{C}$ for $30 \mathrm{~min}$, before TES was added at a final concentration of 
Table 2

Kinetic parameters for 2-OH and 6 $6-\mathrm{OH}$ testosterone hydroxylation and model comparison. ${ }^{\mathrm{a}}$

\begin{tabular}{|c|c|c|c|c|c|c|c|c|c|c|c|}
\hline \multirow[t]{2}{*}{ Enzyme system } & \multirow[t]{2}{*}{ Product } & \multicolumn{4}{|c|}{ Michaelis Menten model } & \multicolumn{5}{|c|}{ Hill Model } & \multirow{2}{*}{$\frac{\text { F-test }}{p \text {-Value }}$} \\
\hline & & $\overline{\mathrm{R}^{2}}$ & $\begin{array}{l}\mathrm{K}_{\mathrm{m}} \\
{[\mu \mathrm{M}]}\end{array}$ & $\begin{array}{l}\mathrm{V}_{\max } \\
\mathrm{pmol}(\mathrm{min} \cdot \mathrm{pmol}) \mathrm{P} 450\end{array}$ & $\begin{array}{l}\mathrm{CL}_{\text {int }}{ }^{\mathrm{b}} \\
\mathrm{ul}(\mathrm{min} \cdot \mathrm{pmol}) \mathrm{P} 450\end{array}$ & $\overline{\mathrm{R}^{2}}$ & $\begin{array}{l}\mathrm{K}^{\prime \mathrm{c}} \\
{[\mu \mathrm{M}]}\end{array}$ & $\begin{array}{l}\mathrm{V}_{\max } \\
\mathrm{pmol}(\mathrm{min} \cdot \mathrm{pmol}) \mathrm{P} 450\end{array}$ & $\begin{array}{l}\mathrm{CL}_{\max }^{\mathrm{d}} \\
\mathrm{ul}(\min \cdot \mathrm{pmol}) \mathrm{P} 450\end{array}$ & $\mathrm{n}^{\mathrm{e}}$ & \\
\hline hСYР3A4 & 6- $\beta-\mathrm{OH}-\mathrm{TES}$ & 0.97 & 18.43 & 61.93 & 3.36 & 0.97 & 20.49 & 64.77 & 3.49 & 134.09 & 0.591 \\
\hline hСYР3A4 & 2-OH-TES & 0.99 & 59.30 & 4.75 & 0.08 & 0.99 & 134.09 & 5.04 & 0.02 & 1.21 & 0.361 \\
\hline eCYP3A95 & 2-OH-TES & 0.97 & 48.00 & 1.97 & 0.04 & 0.98 & 107.30 & 1.78 & 0.01 & 1.55 & 0.412 \\
\hline eCYP3A94 & $6-\beta-O H-T E S$ & 0.96 & 9.32 & 0.25 & 0.03 & 0.96 & 6.48 & 0.27 & 0.09 & 0.73 & 0.780 \\
\hline eСYР3А94 & 2-OH-TES & 0.91 & 21.26 & 0.28 & 0.01 & 0.91 & 30.71 & 0.14 & 0.01 & 0.97 & 0.913 \\
\hline
\end{tabular}

a Data for incubation of testosterone derived from mean values of duplicates.

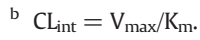

c $\mathrm{K}^{\prime}$ is equivalent to $\mathrm{K}_{\mathrm{m}}$ when $n=1$.

d $\mathrm{CL}_{\max }=\left(\mathrm{V}_{\max } / \mathrm{K}^{\prime}\right)\left[(\mathrm{n}-1) / \mathrm{n}(\mathrm{n}-1)^{1 / \mathrm{n}}\right]$.

e Hill coefficient.

$100 \mu \mathrm{M}$. In accordance to the linear activity obtained with the time-dependent testosterone assay, the different incubation times for CYP3A94, CYP3A95, and CYP3A97 were 79, 38, and 67 min, respectively.

\subsubsection{HPLC and GC/MS}

Testosterone biotransformation was analyzed by high pressure liquid chromatography (HPLC). A detailed description of the device setup has been previously published (Schmitz et al., 2014; Zielinski and Mevissen, 2015). Briefly, the gradient mobile phase consisted of two buffers, containing $39 \%$ methanol, $1 \%$ acetonitrile (buffer A) and $80 \%$ methanol, 2\% acetonitrile (buffer B) and a constant flow rate at $1 \mathrm{ml} / \mathrm{min}$. Furthermore, separation of TES and its metabolites was achieved with an EC 125/4 Lichrospher 100-5 RP-8 column (MacheryNagel, Switzerland). Identification of metabolites was based on comparison of retention times to simultaneously prepared standard samples. All HPLC quantifications were based on normalization of the peak area to the internal standard and calculated by means of linear calibration curves of each metabolite, ranging from 1 to $50 \mu \mathrm{M}$ and TES ranging from 10 to $150 \mu \mathrm{M}$. All calibration curves were analyzed by measuring three independent samples for each standard concentration and were found to be linear $\left(r^{2} \geq 0.99\right)$. All metabolites were confirmed by GC/ MS; the method has been previously described (Schmitz et al., 2014).

\subsection{Statistical analysis}

Graph Pad Prism 6 software (La Jolla, California, USA) was used for the statistical analysis. The linear time was analyzed with a nonlinear regression analysis.

Kinetic parameters were plotted and analyzed by two mathematical models (Michaelis-Menten and Hill) using a nonlinear regression extrasum-of-square F test. The equation that characterized the MichaelisMenten model is

$v=\frac{\mathrm{V} \max [\mathrm{S}]}{\mathrm{Km}+[\mathrm{S}]}$

Briefly, $V$ represents the product formation rate (velocity) and $\mathrm{K}_{\mathrm{m}}$ represents the constant of the concentration of substrate at which the formation rate is half of the $V_{\max } \cdot V_{\max }$ is the maximum formation rate and $[S]$ is the concentration of the substrate.

The alternative Hill model is explained with

$v=\frac{\mathrm{V} \max [\mathrm{S}]^{\mathrm{n}}}{\mathrm{K}^{\prime \mathrm{n}}+[\mathrm{S}]^{\mathrm{n}}}$

where $K^{\prime}$ is the constant of the auto-activation model and it is equivalent to $\mathrm{K}_{\mathrm{m}}$ when $n=1, n$ is represented by the Hill coefficient (Schmitz et al., 2008), (Houston and Kenworthy, 2000). The F-test was used to analyze the fit to the two models.
Standard error of the mean (SEM) and standard deviation (SD) were analyzed on duplicates of two or three independent experiments.

\section{Results}

\subsection{Amplification, cloning and sequencing of equine CYPs, POR, and CYB5}

Amplification and subsequent sequencing of the genes (CYP3A94, CYP3A95, CYP3A97, POR, and CYB5) showed no mutations in CYP3A94, CYP3A97, and CYB5 compared to the annotated equine genes NM_001190939.1, NM_001146164, and NM_001159732 in the EMBL database (NCBI), respectively. CYP3A95 revealed one variation (c.980T > C) with an amino acid change within the same amino acid group when compared to the annotated equine genes NCBI NM 001190940 and NCBI NC_009156.2 (Equus caballus chromosome 13). The obtained equine POR had one variation (c.569G $>$ A) compared to the annotated gene NCBI NM_001122655. However, a comparison of the obtained sequence with equine chromosome 13 (NCBI NC_ 009156.2) using BLAST did not reveal any variation in this region. The CDS of all four genes was successfully cloned into the vector pIEx/Bac1 , and subsequent sequencing of all five enzymes revealed no further mutations or variations.

\subsection{Heterologous expression of equine CYPs}

Five different recombinant viruses were produced and the best expression conditions were found to be MOI 1 and $48 \mathrm{~h}$. Two amplification rounds were necessary to have an appropriate titer to transduct the cells. CYP isoenzymes including a HIS-tag (58 kDa), POR (78 kDa), and CYB5 (16 kDa) were successfully detected by immunoblotting (Fig. 1). The obtained protein size was in accordance with the estimated molecular weight of the respective protein.

CYP levels were measured by spectrophotometry at $450 \mathrm{~nm}$ (Fig. 2) and the amount was calculated for all three CYPs. The reduction capability of POR was detected at $550 \mathrm{~nm}$, and the amount of CYB5 was calculated using the difference between oxidized and reduced protein at $410 \mathrm{~nm}$ and $425 \mathrm{~nm}$, respectively. All five enzymes demonstrated to be functionally active using testosterone as a substrate.

\subsection{Optimal ratios of POR:CYP:CYB5}

The optimal activity of the CYP3A95 was investigated using increasing amounts of POR and CYB5. Different amounts of POR from ratio 2 to 8 were used in combination with different amounts of CYB5. POR to CYP ratios ranging from 2:1 to 8:1 were not significantly different. The same result was found for CYP3A95 to CYB5 ratios. No significant variations in 2-OH-TES production were obtained using different ratios ranging from 1:1 to $1: 2$ (data not shown). Therefore, all three equine CYPs were analyzed with a ratio of 4:1:1 POR:CYP:CYB5. 


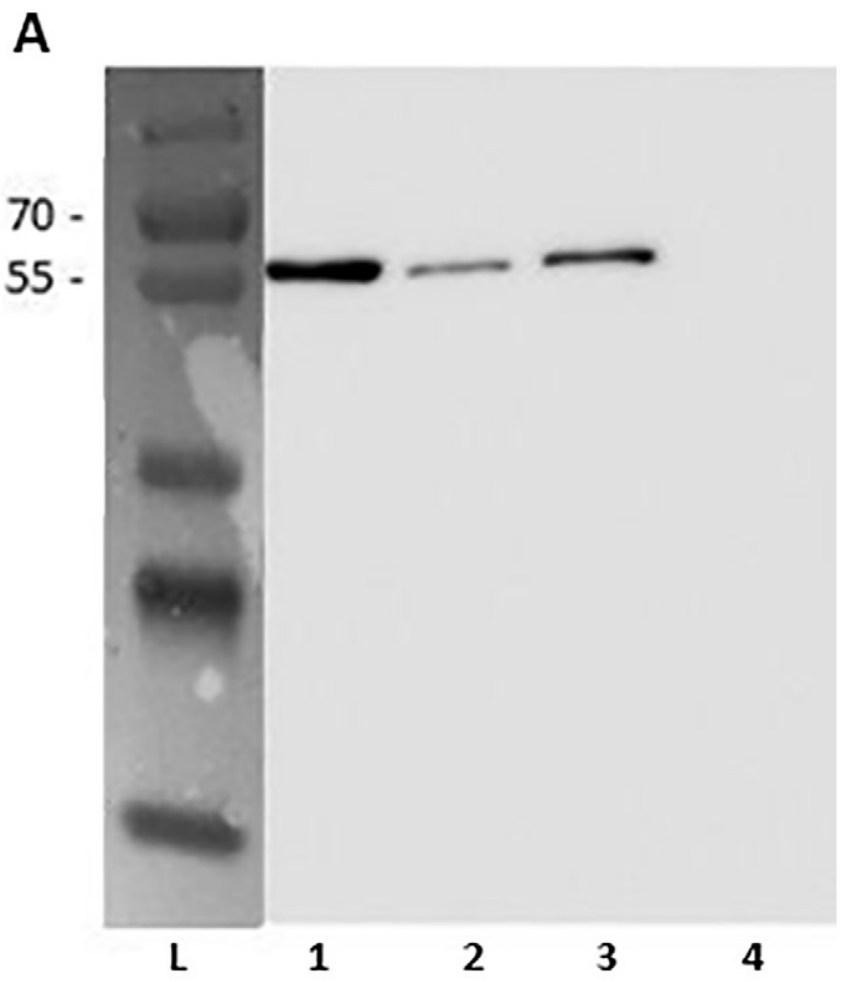

B

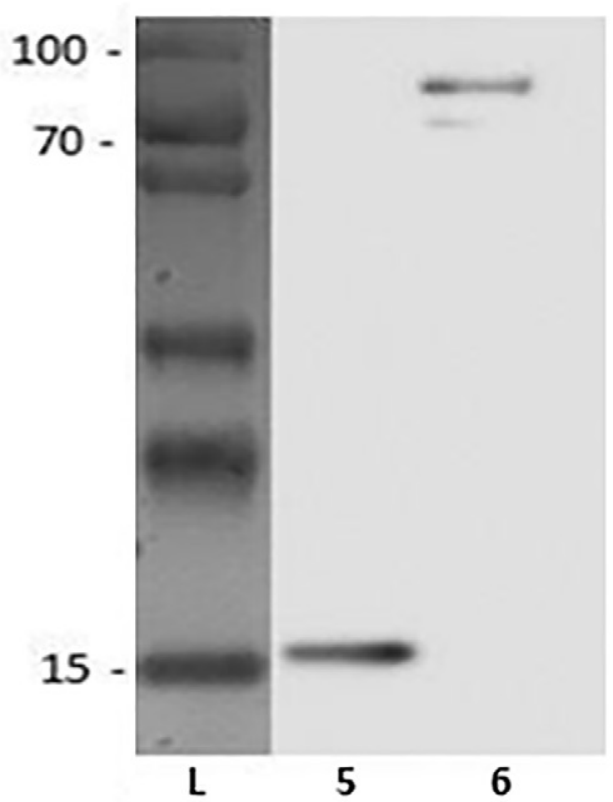

Fig. 1. Western blotting of CYP3A94 (A, column 1), CYP3A95 (A, column 2), CYP3A97 microsomal protein (A, column 3), extracted from transduced Sf9 cells showing a band at $58 \mathrm{kDa}$. Microsomal protein from non-transduced Sf9 samples revealed no band (A, column 4). The band sizes of equine CYB5 (B, column 5 ) and POR (B, column 6 ) are $16 \mathrm{kDa}$ and $78 \mathrm{kDa}$, respectively. $\mathrm{L}=$ ladder $(100,70,55,15 \mathrm{kDa})$; CYP $=$ cytochrome $\mathrm{P} 450, \mathrm{Sf9}=$ spodoptera frugiperda, CYB5 $=$ cytochrome $\mathrm{B} 5, \mathrm{POR}=\mathrm{NADPH}-\mathrm{P} 450$ oxidoreductase.

\subsection{Testosterone metabolism and kinetic activity}

Biotransformation of testosterone was investigated in all three CYPs of the equine $3 \mathrm{~A}$ subfamily using 300 pmol CYP, 300 pmol CYB5 and $1200 \mathrm{pmol}$ POR in a total reaction volume of $1 \mathrm{ml}$. Based on the time

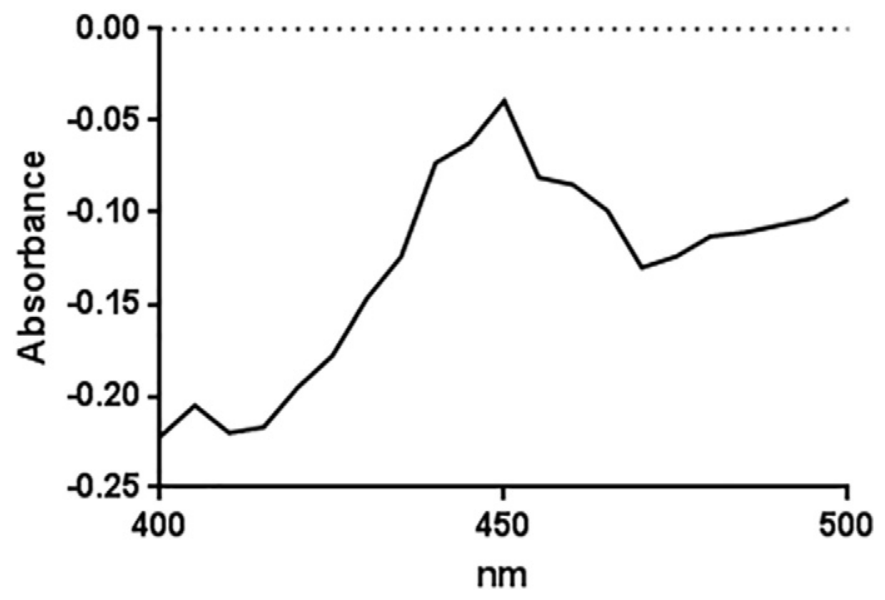

Fig. 2. Carbon Monoxide difference spectrum of the oxidized and the reduced form of the baculovirus-expressed CYP3A94. The spectrum is shown after $1 \mathrm{~min}$ of aeration with carbon monoxide.

dependent experiments, CYP3A95 microsomal preparations were incubated for $38 \mathrm{~min}$, CYP3A94 for $79 \mathrm{~min}$, and CYP3A97 for $67 \mathrm{~min}$ (Fig. 3).

Our results show that $6 \beta-O H-T E S$ was the most prominent metabolite formed by the human CYP3A4 (Fig. 4A). In contrast, the production of $6 \beta-O H-T E S$ varied between the equine CYPs of the $3 A$ subfamily. CYP3A94 produced 6ß-OH-TES, whereas CYP3A95 and CYP3A97 produced traces of $6 \beta-\mathrm{OH}-\mathrm{TES}$ with the levels being close to the detection limit.

Although the differences in the obtained amount, all three equine CYPs oxidized testosterone to 2-OH-TES, with the highest levels being produced by CYP3A95 (Fig. 4D followed by CYP3A94 and CYP3A97.

The kinetic analysis revealed no significant difference in the correlation coefficients $\left(\mathrm{R}^{2}\right)$ between the two models, Michaelis-Menten and Hill. The F-test indicated a better fit for the Michaelis-Menten model compared to the Hill model.

Significant differences between the kinetic parameters $\mathrm{K}_{\mathrm{m}}$ and $\mathrm{V}_{\max }$ were found for the hydroxylation of both metabolites $(6 \beta-O H-T E S$ and 2-OH-TES) (Table 2). $\mathrm{V}_{\max }$ was 13-times higher for 6ß-OH-TES compared to 2-OH-TES in the human CYP3A4, whereas no difference between these metabolites was found in CYP3A94. CYP3A95 produced 7 times more 2-OH-TES compared to CYP3A94. In CYP3A95, the level of $6 \beta-\mathrm{OH}-\mathrm{TES}$ was close to the detection limit, and the kinetic parameters could not be calculated.

Both, the intrinsic and the maximum clearance of $6 \beta-O H-T E S$ were significantly higher in human CYPs compared to all equine CYPs, whereas the clearance was similar in both species for 2-OH-TES (Table 2).

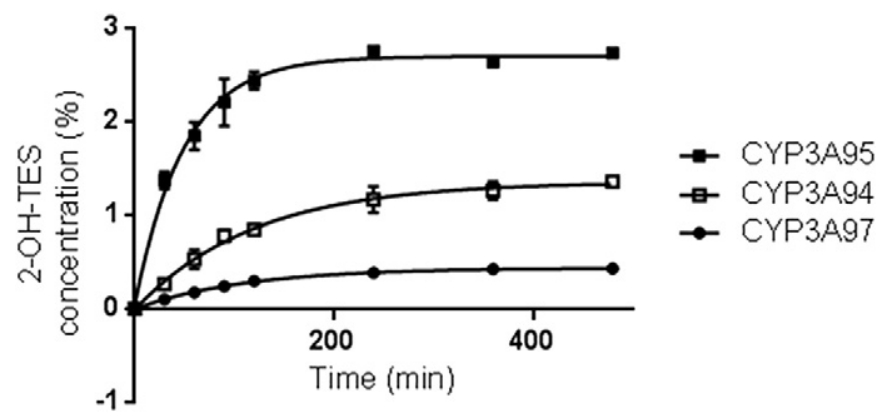

Fig. 3. Testosterone 2-hydroxylation of three different equine isoenzymes of the CYP3A subfamily after incubation with $100 \mu \mathrm{M}$ TES after 30, 60, 90, 120, 240, 360, 480 min; 2OH-TES was most prominent in microsomal protein from CYP3A95 (ם) followed by CYP3A94 $(\square)$ and CYP3A97 (•) 2-OH-TES = 2-hydroxytestosterone. 

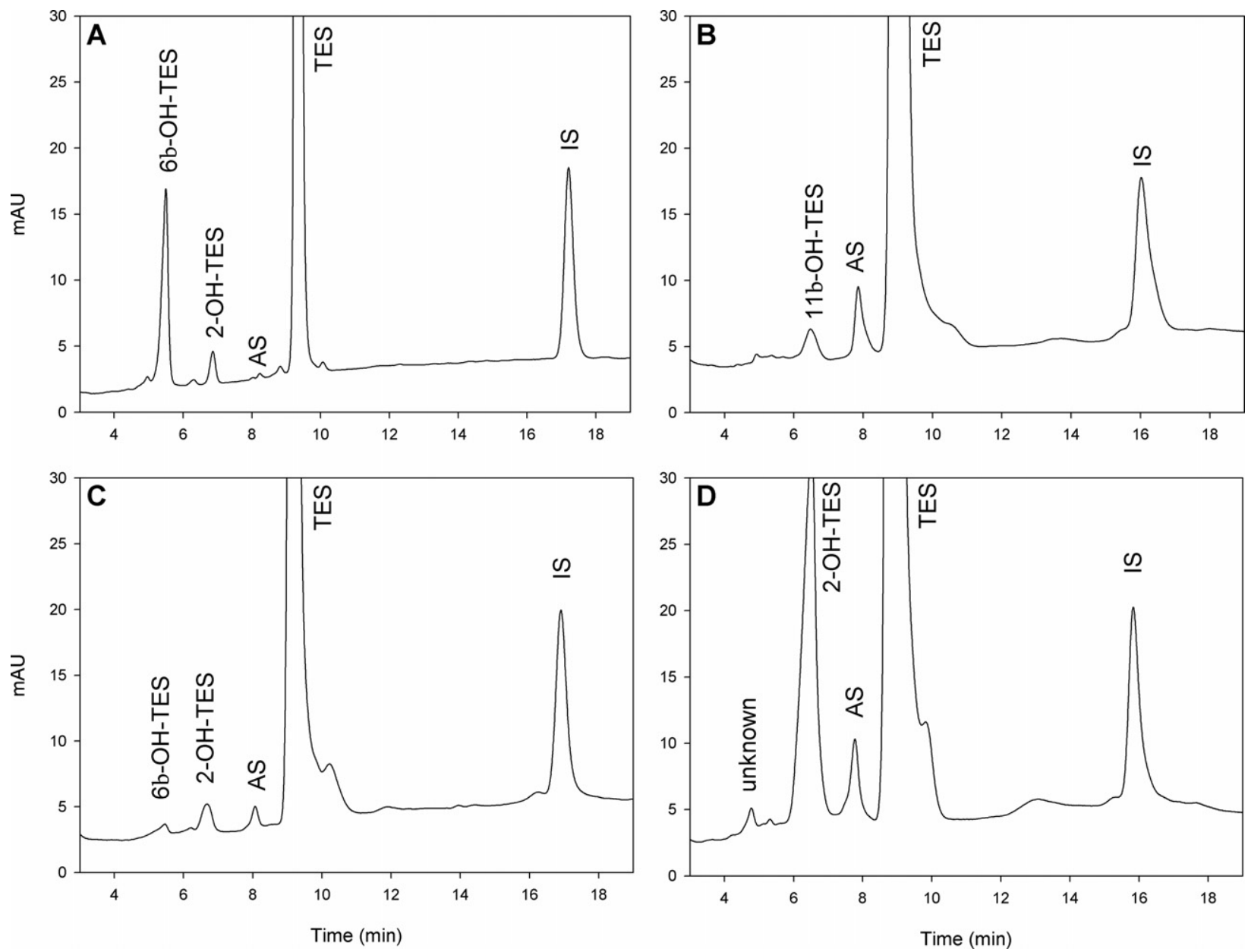

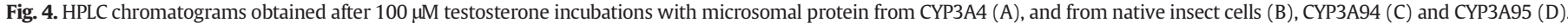

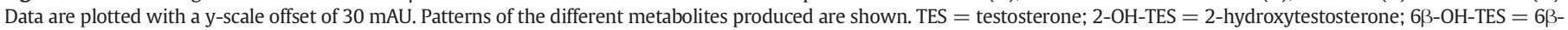
hydroxytestosterone; $11 \beta-O H-T E S=11 \beta$-hydroxytestosterone; AS = androstenedione; IS = internal standard.

As shown in Fig. 4C all three equine CYPs produced androstenedione. Microsomes of native, non-transducted Sf9 cells formed 11ß-OH-TES and androstenedione, but no 63-OH-TES and 2-OH-TES.

\subsection{CYP inhibition}

The metabolism of TES to 6 3 -OH-TES and 2-OH-TES in human CYP3A4 was significantly inhibited by ketoconazole ( $p \leq 0.0001)$ (Fig. $5 \mathrm{~A})$. While 2-OH-TES production was completely inhibited, $6 \beta-\mathrm{OH}-$ TES was diminished by $90 \%$.

In all three equine CYPs, the formation of 2-OH-TES was significantly decreased by ketoconazole as illustrated in Fig. 5B. The rate of 2-OH-TES inhibition varied depending on the equine CYP isoform. A 100\% inhibition of 2-OH-TES was obtained in CYP3A94 $(p \leq 0.001)$ and CYP3A97 $(p \leq 0.0001)$, whereas the 2-OH-TES inhibition was not complete in CYP3A95 as shown in Fig. 5B $(p \leq 0.001)$.

Besides CYP3A4, only CYP3A94 produced $6 \beta-O H-T E S$. The formation of $6 \beta-\mathrm{OH}-\mathrm{TES}$ was completely inhibited by ketoconazole $(p \leq 0.0001)$ in CYP3A94 (data not shown).

\section{Discussion}

\subsection{Recombinant expression}

In this study, three out of seven equine CYP isoenzymes of the subfamily 3A, namely CYP3A94, CYP3A95, and CYP3A97, and two auxiliary proteins (POR and CYB5) were heterologously expressed in insect cells
(Sf9 cells). In contrast to rat, human, and some canine single CYPs, equine single CYPs are not commercially available. Baculovirus technology and Sf9 cells are often used for the expression of CYPs since they are able to produce high yields of the favoured recombinant protein. Furthermore, these cells have the ability to conduct eukaryotic post-translational modifications (Demain and Vaishnav, 2009). Post-translational modifications in insect cells are not always the same as in higher eukaryotes regarding the $\mathrm{N}$-glycosylation. Despite the not mammalian like glycosylation, recombinant proteins are successfully expressed in insect cells (Kost et al., 2005; Yu et al., 2016). The Sf9 cells in our study produced high amounts of functionally active equine CYPs, POR, and CYB5. On the other hand, insect cells have CYPs themselves, which may interfere with the recombinant equine CYPs from other species depending on CYP and the substrate investigated.

\subsection{Polymorphisms in equine POR and CYPS}

Equine POR and CYB5 genes were only rarely used in heterologously expressed single CYP experiments. Interestingly, a variation (c.569G > A) was identified when the equine POR sequence from this study was compared to the equine sequence NM_001122655 published by NCBI. Whereas our cDNA was obtained from a Swiss warmblood horse, the published sequence originated from a Swedish standard bred trotters (Tydén et al., 2008). It seems likely that this variation is due to differences between horse breeds. The same variation found in this study was also found in a previous publication with Swiss warmblood and Franches-Montagnes horses (Dettwiler et al., 2014). 


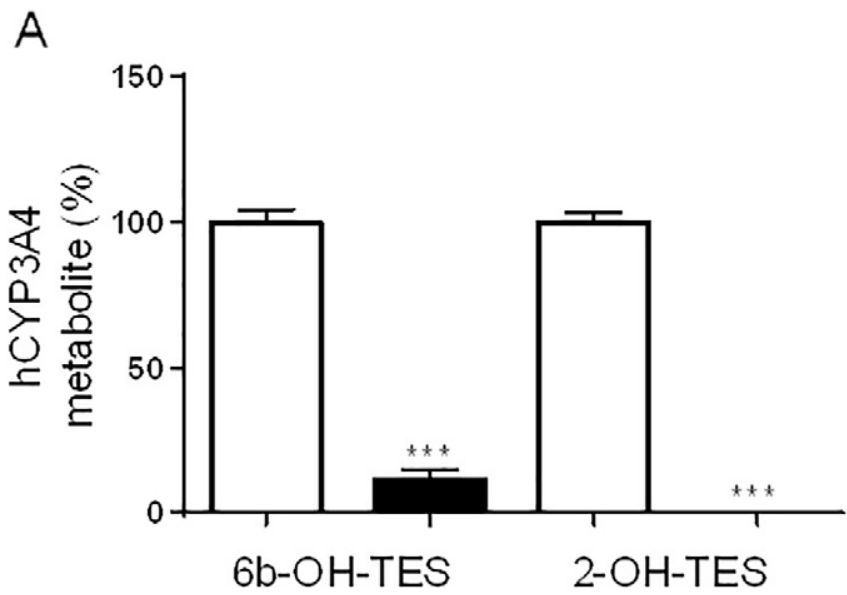

B

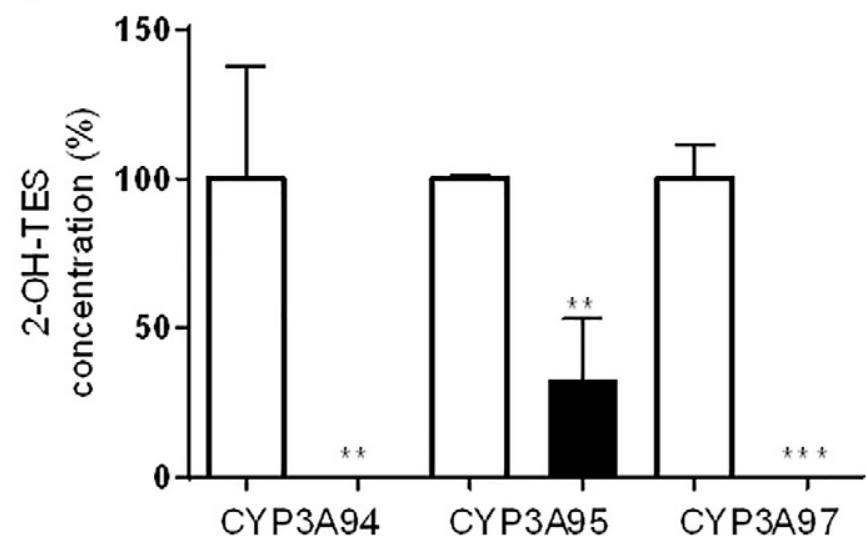

Fig. 5. Ketoconazole inhibition of $6 \beta-\mathrm{OH}-\mathrm{TES}$ and 2-OH-TES formation in the human CYP3A4 (black bars) compared to the non-inhibited control (white bars) (A). Ketoconazole preincubation significantly inhibited 2-OH-TES in equine CYP3A94, CYP3A95 and CYP3A97 (B). Data were recorded in $\mu \mathrm{M}$ metabolite and are shown in percent metabolite formation relative to the native samples (non-inhibited). The bars represent the mean and the SD from three independent experiments. Significant differences are labeled with asterisks; ${ }^{*}=p \leq 0.01 ;{ }^{* *}=p \leq 0.001 ;{ }^{* * *}=p \leq 0.0001$.

These sequences were also identical when blasted to the equine chromosome 13 (NCBI NC_009156.2) originating from an English thoroughbred mare. Further studies are required to investigate which polymorphisms are common and functionally relevant. The obtained POR was found to be highly active in our study.

We also discovered a variation (c.980T $>$ C) in CYP3A95 compared to the NCBI sequence NM_001190940 and to the Equus caballus chromosome 13 (NC_009156.2) (Schmitz et al., 2010a, 2010b). However, CYP3A95 was found to be highly active in our study, and all three isoenzymes including equine POR and CYB5 were successfully expressed in Sf9.

\subsection{Testosterone metabolite formation and kinetics}

All equine CYPs formed 2-OH-TES with the amount being isoenzyme-dependent. This metabolite was formed most prominently by CYP3A95 (Fig. 4D), followed by CYP3A94 and CYP3A97. To our knowledge, this is the first time that 2-OH-TES was demonstrated to be a metabolite of testosterone in horses. Besides $6 \beta-\mathrm{OH}-\mathrm{TES}$ and minor amounts of androstenedione, 2-OH-TES was also detected in CYP3A4. In accordance with our results, $6 \beta-\mathrm{OH}-\mathrm{TES}$ and 2-OH-TES have been reported in TES metabolism in CYP3A4 with 6 $3-\mathrm{OH}-\mathrm{TES}$ being the most prominent metabolite (Usmani et al., 2003). In equine liver samples, $6 \beta-O H-T E S, 11 \beta-O H-T E S$, and androstenedione were obtained, but no 2-OH-TES was found (Schmitz et al., 2014; Zielinski and Mevissen,
2015). The variable expression of all CYPs in liver microsomes and possibly a low activity and a low substrate affinity of all three equine CYPs investigated may explain the absence of this metabolite in equine liver microsomal preparations. As shown in Fig. 4D, CYP3A95 produced only 2-OH-TES with traces of 6 3 -OH-TES at the two highest testosterone concentrations used, whereas CYP3A94 metabolized testosterone to both hydroxylated compounds, 2-OH-TES and 6ß-OH-TES (Fig. 4C), with 2-OH-TES being the main metabolite produced. However, the $\mathrm{V}_{\max }$ values were similar for both metabolites. Therefore, we hypothesize that CYP3A94 catalyzes both reactions at the same time with a faster $6 \beta$-hydroxylation activity. This is in accordance with the estimated $\mathrm{K}_{\mathrm{m}}$ for $6 \beta$-hydroxylation being 2.28 times smaller compared to the $\mathrm{K}_{\mathrm{m}}$ of the 2-hydroxylation of CYP3A94. While the estimated $\mathrm{V}_{\text {max }}$ levels depend on the amount of enzyme present in the in vitro system used, expression levels in the liver and other organs are important. It has been reported that CYPA97 is highly expressed in the liver, followed by CYPs 3A89, 96, 94 and 93 (Tyden et al., 2012). Another study demonstrated highest expression levels CYP3A89 followed by CYP3A 96 and 97 (Knych et al., 2010). Interestingly, individual variability in the CYP expression was $>1000$-fold.

\subsection{Comparison between species}

Whereas testosterone $6 \beta$-hydroxylation has been demonstrated as the main enzyme-specific reaction for CYP3A4 (Yuan et al., 2002), our results showed that 2-OH-TES is the main metabolite produced by members of the equine CYP3A4 subfamily. The analysis of the equine CYPs revealed different velocities in the testosterone hydroxylation rates with 63-hydroxylation catalyzed by CYP3A94, and only traces of this metabolite were detected from CYPs 95 and 97. It has been reported that the equine isoenzymes have a very low isoenzyme-dependent $6 \beta$ hydroxylase activity compared to the human orthologue, which may explain the species differences. Knych et al. (2010) described the metabolism rate for testosterone being 20-times slower in equine CYP3A96 compared to the human orthologue. Different expression systems and purification methods used may explain these differences. The enzymes were resuspended with 50 mM NDSB211, which may have an effect on the activity of the CYPs. We used equine CYB5 instead of rat CYB5 (Knych et al., 2010). This might lead to a different metabolite pattern and hydroxylation activity. Chauret et al. (1997) reported a 7-times slower 63-hydroxylase activity in horses, dogs, and cats. In addition, a similar pattern was seen for diclofenac and dextromethorphan in the CYP2C/D equine subfamilies (DiMaio Knych and Stanley, 2008). Due to the production of androstenedione in native insect cells, the formation of this metabolite in transduced cells with the equine CYPs was not quantifiable. It has been demonstrated that insects express CYPs for physiological functions and metabolism of xenobiotics (Schuler and Berenbaum, 2013). Testosterone hydroxylation has been described in the house fly CYP6A1 (Murataliev et al., 2008). Sf9 cells are ovary cells, and therefore it is likely that they metabolize testosterone.

\subsection{POR, CYP, and CYB5 ratios}

Different ratios of POR:CYP:CYB5 were used in order to investigate the effect of the equine POR on the functional CYP activity using testosterone as substrate. Since there were no significant differences found, the ratio of 4:1 (POR:CYP) was used to allow a comparison to previously published data (Knych et al., 2010). CYB5 is known to be important for the function of several CYPs, especially in human CYP3A4, where it serves as an electron supplier for CYP function, and has also several other functions (Nakajima et al., 2002; Schenkman and Jansson, 2003). Several CYB5 mutations have been reported to significantly affect the CYP3A4 activity (Peng and Auchus, 2014). Since equine POR and CYB5 are not commercially available, human or rat POR and CYB5 are often used in in vitro systems (Knych et al., 2010). We cloned and expressed equine POR and CYB5, and therefore, results cannot be 
directly compared. It has also been suggested that the amount of the auxiliary proteins depend on the substrate (Schenkman and Jansson, 2003).

\subsection{CYP inhibition}

Ketoconazole, a potent reversible inhibitor of human CYP3A4 (Greenblatt, 2014), was used to analyze the inhibition of the equine CYP3A isoforms on the testosterone metabolite formation. Previous studies from our laboratory showed that ketoconazole was demonstrated to completely inhibit ketamine- $N$-demethylation in single human CYP3A4 (Mössner et al., 2011). Furthermore, it was reported that ketoconazole $(5 \mu \mathrm{M})$ was able to completely eliminate the formation of $6 \beta-$ OH-TES in human liver microsomes (Newton et al., 1995).

To our knowledge, there are no published data concerning the inhibition of 2-OH-TES metabolite in the equine CYP3A4 isoforms. Ketoconazole resulted in a complete (CYP3A94 and CYP3A97) or almost complete inhibition (CYP3A95) for 2-OH-TES. Similar to the inhibition of 6B-hydroxylation in CYP3A4, a $100 \%$ inhibition was seen in CYP3A94. These findings indicate similar substrate specificity in equine compared to human CYPs of the $3 \mathrm{~A}$ subfamily.

\section{Conclusion}

Equine CYPs 3A94, 3A95, and 3A97 were found to be active after heterologous expression in insect cells. Substantial species differences and variations between isoenzymes of one subfamily resulted in similar metabolite patterns but differences in the amount of metabolites and in the hydroxylation activity. Characterization of equine CYPs allows mapping their involvement in the metabolism of drug and further enables predictions on possible drug-drug interactions. Further research on the involvement of CYPs of the $3 \mathrm{~A}$ subfamily using drugs use in equine therapy is necessary.

\section{Conflict of interest statement}

The authors declare no conflict of interest.

\section{Transparency document}

The Transparency document associated with this article can be found, in online version.

\section{Acknowledgements}

The authors thank Bernhard Dick for the GC-MS analysis and Sascha Beneke for providing the Sf9 cells. We also thank Friederike Sandbaumhüter for helping with the analysis of the pharmacokinetic data. The authors acknowledge the financial support from the Swiss National Science Foundation [No. 310030_149954].

\section{References}

Bohnert, T., Patel, A., Templeton, I., Chen, Y., Lu, C., Lai, G., Leung, L., Tse, S., Einolf, H.J., Wang, Y.H., Sinz, M., Stearns, R., Walsky, R., Geng, W., Sudsakorn, S., He, L., Wahlstrom, J., Keirns, J., Narayanan, R., Lang, D., Yang, X., 2016. Evaluation of a new molecular entity as a victim of metabolic drug-drug interactions - an industry perspective. Drug Metab. Dispos. 44, 1399-1423.

Capponi, L., Schmitz, A., Thormann, W., Theurillat, R., Mevissen, M., 2009. In vitro evaluation of differences in phase 1 metabolism of ketamine and other analgesics among humans, horses, and dogs. Am. J. Vet. Res. 70, 777-786.

Chauret, N., Gauthier, A., Martin, J., Nicoll-Griffith, D.A., 1997. In vitro comparison of cytochrome P450-mediated metabolic activities in human, dog, cat, and horse. Drug Metab. Dispos. 25, 1130-1136.

Demain, A.L., Vaishnav, P., 2009. Production of recombinant proteins by microbes and higher organisms. Biotechnol. Adv. 27, 297-306.

Dettwiler, R., Schmitz, A.L., Plattet, P., Zielinski, J., Mevissen, M., 2014. Heterologous expression of equine CYP3A94 and investigation of a tunable system to regulate coexpressed NADPH P450 oxidoreductase levels. PLoS One 9, e113540.
DiMaio Knych, H.K., Stanley, S.D., 2008. Complementary DNA cloning, functional expression and characterization of a novel cytochrome P450, CYP2D50, from equine liver. Biochem. Pharmacol. 76, 904-911.

FDA, 2006. Guidance for Industry: Drug Interaction Studies - Study Design, Data Analysis, and Implications for Dosing and Labeling. Food and Drug Administration, Rockville, $\mathrm{MD}$, pp. 1-40.

Fink-Gremmels, J., 2008. Implications of hepatic cytochrome P450-related biotransformation processes in veterinary sciences. Eur. J. Pharmacol. 585, 502-509.

Gan, L., von Moltke, L.L., Trepanier, L.A., Harmatz, J.S., Greenblatt, D.J., Court, M.H., 2009. Role of NADPH-cytochrome P450 reductase and cytochrome-b5/NADH-b5 reductase in variability of CYP3A activity in human liver microsomes. Drug. Metab. Disp. 37 (1), 90-96.

Greenblatt, D.J., 2014. The ketoconazole legacy. Clin. Pharmacol. Drug Dev. 3, 1-3.

Guengerich, F., 2003. Cytochromes P450, drugs, and diseases. Mol. Interv. 3, 194-204.

Houston, J.B., Kenworthy, K.E., 2000. In vitro-in vivo scaling of CYP kinetic data not consistent with the classical Michaelis-Menten model. Drug Metab. Dispos. 28, 246-254.

Khalil, W.F., Saitoh, T., Shimoda, M., Kokue, E., 2001. In vitro cytochrome P450-mediated hepatic activities for five substrates in specific pathogen free chickens. J. Vet. Pharmacol. Ther. 24, 343-348.

Knych, H.K., DeStefano, Shields C., Buckpitt, A.R., Stanley, S.D., 2009. Equine cytochrome P450 2C92: cDNA cloning, expression and initial characterization. Arch. Biochem. Biophys. 485, 49-55.

Knych, H.K., McKemie, D.S., Stanley, S.D., 2010. Molecular cloning, expression, and initial characterization of members of the CYP3A family in horses. Drug Metab. Dispos. 38 1820-1827.

Kost, T.A., Condreay, J.P., Jarvis, D.L., 2005. Baculovirus as versatile vectors for protein expression in insect and mammalian cells. Nat. Biotechnol. 23, 567-575.

Li, T., Apte, U., 2015. Bile acid metabolism and signaling in cholestasis. Inflamm. Cance 263-302.

Mössner, L., Schmitz, A., Theurillat, R., Thormann, W., Mevissen, M., 2011. Inhibition of cytochrome P450 enzymes involved in ketamine metabolism by use of liver microsomes and specific cytochrome P450 enzymes from horses, dogs, and humans. Am. J. Vet. Res. 72, 1505-1513.

Murataliev, M., Guzov, V., Walker, F., Feyereisen, R., 2008. P450 reductase and cytochrome b5 interactions with cytochrome P450: effects on house fly CYP6A1 catalysis. Insect Biochem. Mol. Biol. 38, 1008-1015.

Nakajima, M., Tane, K., Nakamura, S., Shimada, N., Yamazaki, H., Yokoi, T., 2002 Evaluation of approach to predict the contribution of multiple cytochrome P450s in drug metabolism using relative activity factor: effects of the differences in expression levels of NADPH-cytochrome P450 reductase and cytochrome b(5) in the expression system and the differences in the marker activities. J. Pharm. Sci. 91, 952-963.

Nakayama, S.M., Ikenaka, Y., Hayami, A., Mizukawa, H., Darwish, W.S., Watanabe, K.P., Kawai, Y.K., Ishizuka, M., 2016. Characterization of equine cytochrome P450: role of CYP3A in the metabolism of diazepam. J. Vet. Pharmacol. Ther. 39, 478-487.

Nebbia, C., Dacasto, M., Rossetto Giaccherino, A., Giuliano Albo, A., Carletti, M., 2003. Comparative expression of liver cytochrome P450-dependent monooxygenases in the horse and in other agricultural and laboratory species. Vet. J. 165, 53-64.

Newton, D.J., Wang, R.W., Lu, A.Y., 1995. Cytochrome P450 inhibitors. Evaluation of specificities in the in vitrometabolism of therapeutic agents by human liver microsomes. Drug Metab. Dispos. 23, 154-158.

Novotná, A., Krasulová, K., Bartoňková, I., Korhoňová, M., Bachleda, P., Anzenbacher, P., Dvořák, Z., 2014. Dual effects of ketoconazole cis-enantiomers on CYP3A4 in human hepatocytes and HepG2 cells. PLoS One 9, e111286.

Omura, T., Sato, R., 1964. The carbon monoxide-binding pigment of liver microsomes. J. Biol. Chem. 239, 2370-2385.

Pandey, A.V., Flück, C.E., 2013. NADPH P450 oxidoreductase: structure, function, and pathology of diseases. Pharmacol. Ther. 138, 229-254.

Peng, H.-M., Auchus, R.J., 2014. Two surfaces of cytochrome b5 with major and minor contributions to CYP3A4-catalyzed steroid and nifedipine oxygenation chemistries. Arch. Biochem. Biophys. 541, 53-60.

Scarth, J.P., Teale, P., Kuuranne, T., 2011. Drug metabolism in the horse: a review. Drug Test. Anal. 3, 19-53.

Schenkman, J.B., Jansson, I., 2003. The many roles of cytochrome b5. Pharmacol. Ther. 97, 139-152.

Schmitz, A., Portier, C.J., Thormann, W., Theurillat, R., Mevissen, M., 2008. Stereoselective biotransformation of ketamine in equine liver and lung microsomes. J. Vet Pharmacol. Ther. 31, 446-455.

Schmitz, A., Demmel, S., Peters, L.M., Leeb, T., Mevissen, M., Haase, B., 2010a. Comparative human-horse sequence analysis of the CYP3A subfamily gene cluster. Anim. Genet $41,72-79$.

Schmitz, A., Thormann, W., Moessner, L., Theurillat, R., Helmja, K., Mevissen, M., 2010b. Enantioselective CE analysis of hepatic ketamine metabolism in different species in vitro. Electrophoresis 31, 1506-1516.

Schmitz, A., Zielinski, J., Dick, B., Mevissen, M., 2014. In vitro metabolism of testosterone in the horse liver and involvement of equine CYPs 3A89, 3A94 and 3A95. J. Vet. Pharmacol. Ther. 37, 338-347.

Schuler, M.A., Berenbaum, M.R., 2013. Structure and function of cytochrome P450S in insect adaptation to natural and synthetic toxins: insights gained from molecular modeling. J. Chem. Ecol. 39, 1232-1245.

Shou, M., Norcross, R., Sandig, G., Lu, P., Li, Y., Lin, Y., Mei, Q., Rodrigues, A.D., Rushmore, T.H., 2003. Substrate specificity and kinetic properties of seven heterologously expressed dog cytochromes p450. Drug Metab. Dispos. 31, 1161-1169.

Stefanski, A., Mevissen, M., Moller, A.M., Kuehni-Boghenbor, K., Schmitz, A., 2013. Induction of cytochrome P450 enzymes in primary equine hepatocyte culture. Toxicol. In Vitro 27, 2023-2030. 
Thompson, C.M., Montes, J., Aucoin, M.G., Kamen, A.A., 2016. Recombinant protein production in large-scale agitated bioreactors using the baculovirus expression vector system. Methods Mol. Biol. 1350, 241-261.

Tyden, E., Olsen, L., Tallkvist, J., Tjalve, H., Larsson, P., 2008. Cytochrome P450 3A, NADPH cytochrome $\mathrm{P} 450$ reductase and cytochrome b5 in the upper airways in horse. Res. Vet. Sci. 85, 80-85.

Tydén, E., Olsén, L., Tallkvist, J., Tjälve, H., Larsson, P., 2008. Cytochrome P450 3A, NADPH cytochrome P450 reductase and cytochrome b5 in the upper airways in horse. Res. Vet. Sci. 85, 80-85.

Tyden, E., Lofgren, M., Pegolo, S., Capolongo, F., Tjalve, H., Larsson, P., 2012. Differential gene expression of CYP3A isoforms in equine liver and intestines. J. Vet. Pharmacol. Ther.

Usmani, K., Rose, R., Hodgson, E., 2003. Inhibition and activation of the human liver microsomal and human cytochrome P450 3A4 metabolism of testosterone by deploymentrelated chemicals. Drug Metab. Dispos. 31, 384-391.

Venkatakrishnan, K., von Moltke, L., Court, M., Harmatz, J., Crespi, C., Greenblatt, D., 2000 Comparison between cytochrome P450 (CYP) content and relative activity approaches to scaling from cDNA-expressed CYPs to human liver microsomes: ratios of accessory proteins as sources of discrepancies between the approaches. Drug Metab. Dispos. 28, 1493-1504.

van Waterschoot, R.A., Schinkel, A.H., 2011. A critical analysis of the interplay between cytochrome P450 3A and P-glycoprotein: recent insights from knockout and transgenic mice. Pharmacol. Rev. 63, 390-410.
Werk, A.N., Cascorbi, I., 2014. Functional gene variants of CYP3A4. Clin. Pharmacol. Ther. 96, 340-348.

Wilkinson, G.R., 2005. Drug metabolism and variability among patients in drug response. N. Engl. J. Med. 352, 2211-2221.

Yao, M., Dai, M., Liu, Z., Huang, L., Chen, D., Wang, Y., Peng, D., Wang, X., Liu, Z., Yuan, Z., 2011. Comparison of the substrate kinetics of pig CYP3A29 with pig liver microsomes and human CYP3A4. Biosci. Rep. 31, 211-220.

Yu, J., Ritchie, T.K., Mulgaonkar, A., Ragueneau-Majlessi, I., 2014. Drug disposition and drug-drug interaction data in 2013 FDA new drug applications: a systematic review. Drug Metab. Dispos. 42, 1991-2001.

Yu, K., Yu, Y., Tang, X., Chen, H., Xiao, J., Su, X.-D., 2016. Transcriptome analyses of insect cells to facilitate baculovirus-insect expression. Protein Cell 7, 373-382.

Yuan, R., Madani, S., Wei, X., Reynolds, K., Huang, S., 2002. Evaluation of cytochrome p450 probe substrates commonly used by the pharmaceutical industry to study in vitro drug interactions. Drug Metab. Dispos. 30, 1311-1319.

Zielinski, J., Mevissen, M., 2015. Inhibition of in vitro metabolism of testosterone in human, dog and horse liver microsomes to investigate species differences. Toxicol. In Vitro 29, 468-478. 